\title{
ON A THEOREM OF RICHARDSON
}

\section{J. R. ISBELL ${ }^{1}$}

Given a set $S$ with an irreflexive relation $>$, define the operators dom and max as follows. For any subset $T$ of $S$, dom $T$ is the set of all $x$ in $S$ such that for some $t$ in $T, t>x ; \max T=T$-dom $T$. A solution of $(S,>)$ is a subset $T$ of $S$ satisfying $T=S-\operatorname{dom} T$.

A solution of an $n$-person game in the sense of [3] is precisely a solution, as just defined, of a certain system $(S,>)$ associated with the game. Von Neumann and Morgenstern investigated various combinatorial criteria for solutions in [3, pp. 587-602]. Richardson has given further criteria; in particular, in [4] he established that if every unoriented cycle in $S$ is even then $S$ has a solution. ${ }^{2}$ An unoriented cycle is a subset $\left(x_{1}, \cdots, x_{n}\right)$ of $S$ such that for $i=1, \cdots, n-1$, either $x_{i}>x_{i+1}$ or $x_{i+1}>x_{i}$, and also $x_{1}>x_{n}$ or $x_{n}>x_{1}$. This note establishes a reduction for general $(S,>)$ which yields a simplified proof of Richardson's theorem. The reduction is an elaboration of one which has been studied by Gillies [1].

Obviously any solution $T$ must contain max $S$, since every point is in $T$ or in dom $T$. Hence no solution can contain any point of dom max $S$, since no point of $T$ is in dom $T$. Inductively let $S_{1}=S, M_{\alpha}=\max S_{\alpha}$, $D_{\alpha}=\operatorname{dom} M_{\alpha}, S_{\alpha+1}=S_{\alpha}-M_{\alpha}-D_{\alpha} ;$ and for limit ordinals $\alpha$, let $S_{\alpha}=\bigcap\left[S_{\beta} \mid \beta<\alpha\right]$. By induction, any solution must contain $M=\bigcup M_{\alpha}$ and be disjoint from $D=\cup D_{\alpha}$. (Gillies studied conditions for $M_{1}$ to be a solution [1].)

Consider the set $R=S-M-D$. The set $\operatorname{dom} M=D$ is disjoint from $R$. Therefore for any subset $T$ of $S-D$, dom $T \cap R$ $=\operatorname{dom}(T \cap R) \cap R$. Thus (1) if $T$ is a solution of $(S,>)$ then $T \cap R$ is a solution of $(R,>)$, and (2) if $T \cap R$ is a solution of $(R,>)$ then $(T \cap R) \cup M$ is a solution of $(S,>)$. This establishes a one-to-one correspondence between solutions of $R$ and solutions of $S$.

By construction of $M, \max R$ is empty. Therefore solving $S$ reduces to solving $R$, where max $R$ is empty. In Richardson's case this is done by a theorem of König [2]: if all unoriented cycles in $R$ are even, then $R$ can be "colored in two colors," i.e. partitioned into two sets, $P, Q$, such that $x>y$ never holds if $x$ and $y$ are both in $P$ or both in $Q$.

Received by the editors February 1, 1957.

1 Supported by the Office of Naval Research and the National Science Foundation.

${ }^{2}$ Richardson assumed the relation $>$ to be antisymmetric, but this hypothesis is not used (as Richardson points out). 
This implies $\operatorname{dom} P$ is a subset of $Q$, and since $\max R$ is empty, dom $P=Q$. Thus $P \cup M$ is a solution of $(S,>)$.

\section{REFERENCES}

1. D. Gillies, Some theorems on n-person games, Thesis, Princeton, 1953.

2. D. König, Uber Graphen und ihre Anwendung auf Determinantentheorie und Mengenlehre, Math. Ann. vol. 77 (1916) pp. 453-465.

3. J. von Neumann and O. Morgenstern, Theory of games and economic behavior, 2d ed., Princeton, 1947.

4. M. Richardson, On weakly ordered systems, Bull. Amer. Math. Soc. vol. 52 (1946) pp. 113-116.

TULANE UNIVERSITY AND

The Institute for Advanced Study

\section{SUBALGEBRAS OF AN ALGEBRA WITH A SINGLE GENERATOR ARE FINITELY GENERATED}

DAVID GALE

In order to prove the assertion above it clearly suffices to consider only the free algebra on one generator and hence the polynomials in one variable over a field $F$. The fact that the sub-algebras of $F[x]$ are all finitely generated is perhaps not surprising, but the author has not been able to discover any proof of it in the literature. (Incidentally, the corresponding statement with the field $F$ replaced by the ring of integers is not true, for the ring of all integral polynomials with even coefficients is clearly not finitely generated.) The proof, though quite simple, would seem to place the problem outside the category of elementary exercises since the solution, at least as presented here, uses some "theory," namely the ever useful facts about modules over principal ideal rings.

We shall prove a somewhat stronger result, namely

Theorem. Let $A \neq F$ be a subalgebra of $F[x]$ and let $n$ be the degree of the polynomial of smallest positive degree in $A$. Then $A$ can be generated by a set of not more than $n+1$ elements.

Proof. We consider first the case $F \subset A$. Let $p$ be a polynomial in $A$ of degree $n$, and let $F[p]$ denote the algebra generated by $p$ and $F$ in $F[x]$. We assert that $F[x]$ is a module over $F[p]$ with generators $1, x, \cdots, x^{n-1}$, for if $q \in F[x]$ we show by induction on the degree of

Received by the editors August 11, 1956. 